Soc. Geogr. Discuss., 3, 183-210, 2007

www.soc-geogr-discuss.net/3/183/2007/

(c) Author(s) 2007. This work is licensed

under a Creative Commons License.

\title{
Raumbezogene Vorstellungsbilder am Beispiel Leipziger Wohnquartiere - ein Annäherungsversuch auf der Grundlage einer visuellen Methodik
}

K. Wiest ${ }^{1}$

${ }^{1}$ Leibniz-Institut für Länderkunde e.V., Schongauerstr. 9, 04329 Leipzig, Germany

Received: 7 November 2006 - Accepted: 10 May 2007 - Published: 23 May 2007

Correspondence to: K. Wiest (k_wiest@ifl-leipzig.de)

Title Page

Abstract

Introduction

Conclusions

References

Tables

Figures

14

4

$>$

Back

Full Screen / Esc

Printer-friendly Version

Interactive Discussion 


\section{Zusammenfassung}

Das Interesse an sozialen Repräsentationen und kulturellen Deutungen des Raumes in der Geographie und Stadtforschung hat auch einen neuen Blick auf Stadtentwicklungsprozesse hervorgebracht. So sind neben der Analyse physisch-materieller 5 Strukturen Fragen nach der symbolischen Konstitution des Raumes, den Facetten des third space (Soja, 1996) in den Vordergrund gerückt. Damit zeigt sich eine stärkere Hinwendung zu kulturellen Aspekten im Sinn fortlaufender gesellschaftlicher Aushandlungsprozesse, um sozialräumliche Strukturen und Verhältnisse zu erklären (u.a. Mills, 1993; Lees, 2004; Ley, 1996, Caulfield, 1989; Lang, 1998; Best, Gebhard, 2001). Re-

10 sidentielle Segregation und Gentrification können in diesem Zusammenhang sowohl als Folgen als auch Gegenstand sozialer Verräumlichungen thematisiert werden, das heißt im Sinn von gesellschaftlichen Bedeutungen und Kategorien, die Orten zugewiesen werden. Geht man von entsprechenden Perspektiven aus, dann findet sich in den Städten der neuen Bundesländer ein interessantes Terrain für die Erforschung der 15 symbolischen Konstruktion von Orten und gesellschaftlichen Wertzuweisung an den Raum. Hier hat der Übergang in ein neues Gesellschaftssystem zu einer besonders raschen Neu- bzw. Umbewertungen räumlicher Strukturen geführt, die unmittelbar die Frage nach der Bedeutung von Verräumlichung als Medium sozialer und kultureller (Neu-)Ordnung und Strukturierung aufwerfen. In diesem Zusammenhang werden im 20 Rahmen des folgenden Beitrags raumbezogene Vorstellungsbilder und symbolische Zuweisungen an Stadtgebiete als Bezugssysteme sozialer Orientierung thematisiert. Am Beispiel von citynahen Altbaugebieten in der Stadt Leipzig werden raumbezogene Bedeutungen und Wertvorstellungen im Alltag der Bewohner und im fachlichen Diskurs der Immobilienbranche als zwei unterschiedliche Aspekte der gesellschaftlichen 25 Produktion des Raumes angesprochen:

- Ausgehend von Tiefeninterviews mit Bewohnern wird der Frage nachgegangen, welche gesellschaftlichen Werte (z.B. Freiheit, Sicherheit) und identitätsstiftenden Kategorien mit bestimmten Stadträumen verbunden werden. Als methodisches
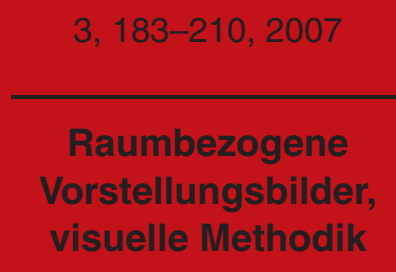

Title Page

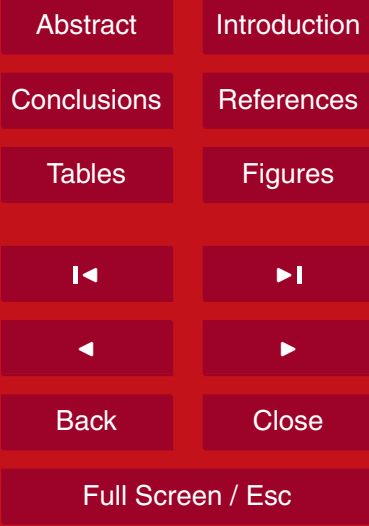

Printer-friendly Version Interactive Discussion 
Hilfsmittel um sich raumbezogenen Vorstellungsinhalten anzunähern, wurden Bilder aus Werbekampagnen benutzt. Dabei wurde davon ausgegangen, dass Werbung gesellschaftlich relevante Strukturen, Sehnsüchte und Identitäten abbildet, die im alltäglichen Leben auch auf Räume projiziert werden.

- Auf der Grundlage von Informations- und Werbematerialen sowie Gesprächen mit Immobilienmaklern werden Repräsentationen des Raumes aufgedeckt, die innerhalb fachlicher Diskurse kommuniziert werden. Im Mittelpunkt steht hier die Frage, wie einzelne Wohngebiete durch die Immobilienbranche zum Zweck der Vermarktung präsentiert werden.

10 Damit stehen Visualisierungen als ein methodisches Instrument der empirischen Forschung im Vordergrund. Ziel war es, sich bildhaft mentalen Repräsentationen bzw. inneren Bildern über das Hilfskonstrukt visuell kommunikativer (Werbe-)Bilder anzunähern. Im Zusammenhang mit Fragen nach raumbezogenen Vorstellungen erscheint Bildmaterial als Bestandteil der empirischen Methodik geeignet, da es auf vorkommunikative Bereiche des Erlebens abhebt, und eine starke assoziative, emotionalisierende Wirkung aufweist. Dabei liegt die Besonderheit von Bildern im Unterschied zur Eindeutigkeit der Sprache in den vielen unterschiedlichen Deutungen, die sie in Abhängigkeit von sozialen und individuellen Interpretationskontexten erlangen können (Schelske, 2001:152). Eine Annäherung an raumbezogene Vorstellungen im

20 Sinn alltäglich erlebter Räume der Repräsentation (s. Tabelle 1) die nicht unmittelbar kommuniziert, sondern eher durch Bilder und Symbole vermittelt werden, scheint eher über visuell vermittelte Erzähl- und Bedeutungsstrukturen möglich, wie sie z.B. auch der Werbung zu Grunde liegen.

Um sozialräumliche Differenzierung zunächst durchgängig als soziales Phänomen 25 zu konzeptionalisieren wird nachfolgend kurz auf Lefebvres Theorie der Produktion des Raumes eingegangen, die die Kategorien „Stadt“ und „Raum“ systematisch in eine übergreifende Gesellschaftheorie integriert (Schmid, 2005:9).
SGD

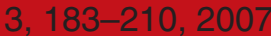

Raumbezogene Vorstellungsbilder visuelle Methodik

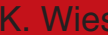

Title Page 


\section{Sozialräumliche Strukturen, raumbezogene Vorstellungsbilder und öffentli- che Diskurse - der Ruf nach integrativen Konzepten in der Stadtforschung}

Sozialräumliche Differenzierung und die gesellschaftliche Auf- und Abwertung städtischer Teilräume eröffnet ein breites Spektrum an theoretischen und ideologischen

5 Erklärungszugängen. Beispiele für die Vielzahl an Betrachtungsweisen dieses Untersuchungsfeldes sind u.a. Analysen des Nachbarschaftswandels, die Beleuchtung der veränderten Nachfrage nach Wohnraum als Ausdruck spezifischer Lebensstil- und Konsumpräferenzen oder als Konsequenz eines Wandels des Nachfrageverhaltens vor dem Hintergrund gesamtökonomischer Veränderungen, (Re-)Investitionen in den Woh10 nungsbestand als Resultat der Mechanismen auf dem Immobilien- und Bodenmarkt bis hin zur symbolischen Inwertsetzungen städtischer Teilräume. Die Unmöglichkeit derart unterschiedliche Aspekte in einem in sich geschlossenen, eindeutigen Konzept zu vereinen, führte in der Stadtforschung zu der Frage, inwieweit hier versucht wird, Prozesse unter einem Dach zu subsumieren, die grundsätzlich unterschiedliche Ursachen 15 haben. Auch die Bestrebungen, eine Erklärungsperspektive gegenüber einer anderen als stichhaltiger herauszustellen, wie z.B. durch das Ausspielen von produktionsorientierten gegenüber kulturorientierten Ansätzen in der Gentrificationforschung, konnte letztendlich nicht überzeugen (dazu u.a. Hamnett, 1991; Lees, 2000 u.v.m.). Diese Schwierigkeiten scheinen zum Teil überwindbar, wenn sozialräumliche Differenzierung im Sinn eines mehrdeutigen, interpretativen theoretischen Zugangs durchgängig als Produkt und Medium sozialer Praxis konzeptionalisiert wird (Phillips, 2002). Das Anliegen (Stadt-)Raum aus der jeweiligen Gesellschaft heraus zu erschließen und zu verstehen, verweist dabei auf Lefebvres Theorie der gesellschaftlichen Produktion des Raumes. So korrespondiert z.B. das wachsende Interesse an diskursiven Konstruktionen der Gentrification eng mit Lefebvres Interesse an „räumlichen Repräsentationen“ bzw. der „Repräsentation des Raumes“ (Phillips, 2002:285). Sein dreidimensionales Konzept umfasst nicht nur eine konkrete Materialität, sondern auch ein gedankliches Konzept sowie eine Raumempfindung bzw. ein Raumerleben (Schmid, 2005:313). Ori-

\section{SGD}

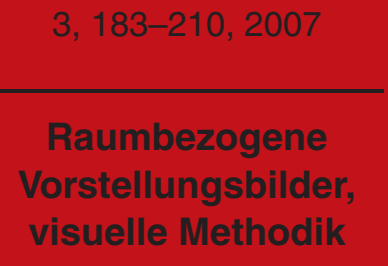

Title Page

Abstract

Introduction

Conclusions

References

Tables

Figures

$1<$

DI

4

$\checkmark$

Back

Close

Full Screen / Esc

Printer-friendly Version

Interactive Discussion 
entiert man sich an diesem Raumverständnis, können sozialräumliche Differenzierungsprozesse unter den Aspekten der materiellen Produktion, der Wissensproduktion und der Bedeutungsproduktion betrachtet werden, die sich gegenseitig beeinflussen, einschränken und überlagern, die sich aber letztendlich nicht völlig in Übereinstim5 mung bringen lassen. In diesem Sinn wäre sozialräumliche Differenzierung nicht als geschlossenes Konzept zu behandeln, sondern im Sinn unterschiedlicher, sich zum Teil ergänzender Zugänge, die auch dem Aspekt der symbolischen Inwertsetzung von Räumen Rechnung tragen. Differenz ist dabei selbst als eine gesellschaftlich produzierte Größe anzusehen, als ein Interpretationsraster mit dessen Hilfe Raum katego10 risiert und bewertet wird (Shields, 1991). Sozialräumlichen Differenzierungen können folgende Prozesse gesellschaftlicher Raumproduktion zugrunde liegen (s. Tabelle 1):

a) die materielle Produktion des Raumes, die eine räumliche Praxis und damit auch einen wahrnehmbaren Aspekt des Raumes herstellt. Lefebvre bezieht sich hier auf die Umgebung, in der der Mensch körperlich im Raum agiert und diesen gestaltet. In un15 serem Untersuchungsbeispiel lässt sich unter anderem thematisieren, welche konkreten materiellen Veränderungen der Raumstrukturen in ostdeutschen Cityrandgebieten nach 1990 durch Investitionen in den Baubestand, durch das Zu- und Wegzugsverhalten bestimmter Bevölkerungsgruppen sowie die Etablierung spezifischer Infrastrukturen stattgefunden haben.

b) die Produktion von Wissen, die theoretische Konzeptualisierungen des Raumes hervorbringt. Diese Repräsentationen des Raumes beziehen sich auf die Ebene fachlicher Diskurse und beinhalten Raumdarstellungen, die gedanklichen Konstrukten entsprechen (Schmid, 2005:216f.). Beispiele wären in unserem Untersuchungszusammenhang der Planungsdiskurs mit der Festlegung und Abgrenzung von Fördergebieten, den Konzepten des Stadtumbaus Ost sowie den städtebaulichen Leitbildern der europäischen bzw. der perforierten Stadt.

c) die Produktion von Bedeutungen, die sich auf einen von den Akteuren erlebten oder gelebten Vorstellungsraum beziehen. Diese Räume der Repräsentation „repräsentieren gesellschaftliche „Werte“ Traditionen, Träume - und nicht zuletzt auch

\section{SGD}

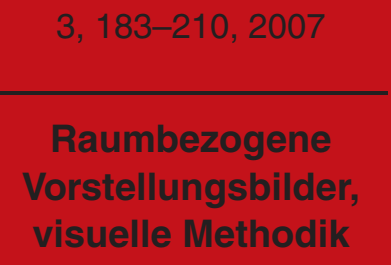

Title Page

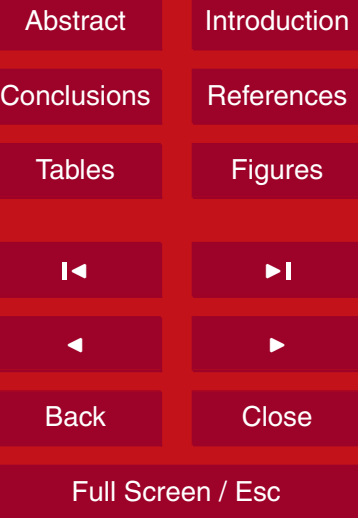

Printer-friendly Version Interactive Discussion 
kollektive Erfahrungen und Erlebnisse“ (Schmid, 2005:223). Diese Formation bezieht sich auf den Raum, wie er durch die mit inm verbundenen Bilder- und Symbolwelten erlebt wird. Er überlagert den realen physischen Raum und setzt seine Gegebenheiten symbolisch um. Beispiele sind im vorliegenden Untersuchungszusammenhang der 5 Mythos von Orten oder raumbezogene Images und Stereotype, die sich auf einen konkreten materiellen Raum beziehen.

Im Folgenden werden einzelne Aspekte dieses dialektischen Raummodells an empirischen Beispielen veranschaulicht. Dabei liegt der Fokus auf der Produktion raumbezogener Vorstellungsinhalte (thirdspace) mit der Frage nach der Übersetzung loka10 ler Gegebenheiten in eine gesellschaftlich geteilte Symbolsprache. Um die Produktion von raumbezogenen Bildern und Symboliken nachvollziehbar zu machen, ist es jedoch zunächst erforderlich, auf die vorhandenen materiellen Strukturen und Gegebenheiten in den Beispielgebieten einzugehen.
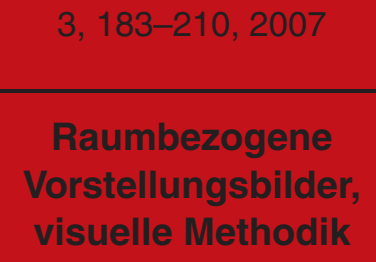

\section{Sozialstruktureller Wandel in Leipzigs Cityrandgebieten seit Mitte der 1990er Jahre}

Die Frage nach der sozialen Interpretation städtischer Strukturen wird im Folgenden am Beispiel citynaher Altbaugebiete in Leipzig behandelt. Hier, wie auch in anderen ostdeutschen Kommunen waren ökonomische und gesellschaftliche Umbrüche nach der Wiedervereinigung Deutschlands mit einer grundlegenden gesellschaftlichen Neubewertung städtischer Teilräume verbunden. Der Frage, welche Werte, sozialen Zuschreibungen und Zugehörigkeiten auf einzelne Stadträume projiziert werden, wird auf der Grundlage von Ergebnissen einer Längsschnittstudie zum Wandel von innenstadtnahen Wohngebieten nachgegangen. ${ }^{1}$ Bei allen drei Gebieten handelt es sich um gründerzeitlich geprägte Wohnquartiere, die in einem Radius von ca. einem Kilometer

\footnotetext{
${ }^{1}$ Grundlage bilden empirische Daten, die in einem 2002 bis 2004 von der DFG geförderten Projekt zum Thema „Segregation und Gentrification in der schrumpfenden Stadt?"erhoben wurden.
}

Title Page

Abstract Introduction

Conclusions References

Tables Figures

1

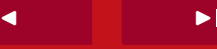

4

$>$

Back

Close

Full Screen / Esc

Printer-friendly Version

Interactive Discussion 
von der Leipziger Innenstadt entfernt liegen. Bauliche Strukturen und die vor diesem Hintergrund eingesetzten Förderinstrumente wiesen bereits Anfang der 1990er Jahre auf unterschiedliche Quartierskarrieren hin (Wiest, Zischner, 2006). Die betrachteten Gebiete lassen sich folgendermaßen charakterisieren:

- Der Neustädter Markt östlich des Leipziger Hauptbahnhofs gelegen, wurde Mitte des 19. Jahrhundert planmäßig als Wohngebiet für die zuwandernde Arbeiterschaft angelegt. In den 1980er Jahren der DDR führten u.a. die Politik des Entmietens und geplante Flächensanierungen zu zunehmenden Abwanderungen und Leerständen, die sich in den ersten Jahren nach der Wiedervereinigung weiter verstärkten. Planungspolitische Antworten auf bauliche Mängel und selektive Abwanderung waren die Ausweisung als Sanierungsgebiet im Jahr 1992 und die Initiierung des Bund-Länder-Programms Soziale Stadt im Leipzig Osten ab 1999.

- Das Waldstraßenviertel liegt nordwestlich des Stadtzentrums zwischen innerstädtischen Grünflächen. Die repräsentative und geschlossene Bebauung des Gebiets stammt im Wesentlichen aus der zweiten Hälfte des 19. Jahrhunderts. Zum Zeitpunkt seiner Entstehung war es ein Quartier des gehobenen Bürgertums und Zentrum des jüdischen Lebens in Leipzig. Heute zählt das Gebiet wieder zu den besten Wohnlagen der Stadt. Dazu beigetragen hat unter anderem der Staus als Fördergebiet des städtebaulichen Denkmalschutzes, der den Bekanntheitsgrad des Viertels Anfang der 1990er Jahre deutlich erhöht hat.

- In der Südvorstadt wird die viertelsprägende gründerzeitliche Blockrandbebauung durch repräsentative freistehende Gebäude sowie Nachkriegsbebauung durchbrochen. Eine relative Nähe zur Grünachse Leipzigs sowie eine, sich seit den 1990er Jahren zunehmend verdichtende gastronomische Infrastruktur bestimmen den Charakter des Gebiets. Der südliche Teil des Untersuchungsraumes ist seit 1994 Sanierungsgebiet.

Ein Vergleich der Sozialstrukturen der Bewohnerschaft seit Mitte der 1990er Jahre hat gezeigt, dass sich in den Leipziger Cityrandgebieten in den letzten zehn Jahren er- 
hebliche Differenzierungen vollzogen haben. Wies die soziale Zusammensetzung der Bewohnerschaft in den drei untersuchten Stadträumen hinsichtlich Alter, Qualifizierung und Einkommen im Jahr 1994 noch eine relativ ähnliche Struktur auf, haben die sozialen Unterschiede zwischen den Gebieten in den nachfolgenden Jahren erheblich 5 zugenommen (Hill, Wiest, 2004:365 ff.; Wiest, Zischner, 2006). So konnte im WaldstraBengebiet ein zunehmender Anteil beruflich etablierter, ökonomisch gesicherter Haushalte festgestellt werden. Dagegen ist am Neustädter Markt seit Mitte der 1990er Jahre eine Zunahme einkommensschwächerer Haushalte und andererseits eine überdurchschnittliche Zuwanderung von Migranten zu beobachten gewesen. Die Südvorstadt hat 10 sich unter der jüngeren Bevölkerung zu einem der gefragtesten Wohngebiete Leipzigs entwickelt. Dies äußert sich unter anderem in einem stark gesunkenem Altersdurchschnitt der Bewohner, unterdurchschnittlichen Raten des Wohnungsleerstands sowie etwas überdurchschnittlichen Mietpreisen auf einzelnen Marktsegmenten.

In Ergänzung zu diesen sichtbaren, materiellen Veränderung der Raumstrukturen, 15 die sich unter anderem in Mobilitätsprozessen und baulichen Maßnahmen (first space, räumliche Praxis) manifestieren, stehen nachfolgend Fragen der gesellschaftlichen Deutung materieller Raumstrukturen im Mittelpunkt, die sozialräumlichen Differenzierungen voraus gehen und auf diese wiederum reagieren. Der Frage nach Zusammenhängen zwischen den Lebensentwürfen der Bewohner und ihrem Bild vom jeweili20 gen Stadtviertel lag die Hypothese zu Grunde, dass auf städtische Teilräume kulturelle Werthaltungen und Vorstellungen von spezifischen Lebensweisen projiziert werden, die nicht zuletzt konkrete Wohnstandortentscheidungen steuern. Damit richtet sich das Interesse auf eine Art symbolischen Code des Raumes, der den Gesellschaftsmitgliedern zum einen den Zugang zu ihrem Raum ermöglicht und zum anderen Zugang zu 25 ihrem Status als Subjekte, als die sie in diesem Raum agieren (Schmid, 2005:225). In Ergänzung dazu wird aufgezeigt, welche raumgebundenen sozialen Zuschreibungen vor dem Hintergrund konkreter Vermarktungsabsichten formuliert werden, denen am Beispiel der Immobilienbranche und der Standortwerbung nachgegangen wird.

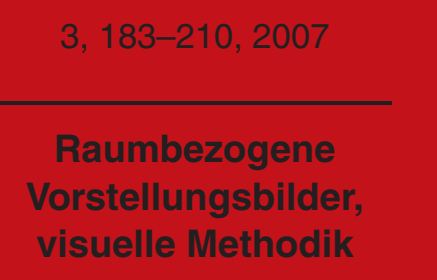

Title Page

Abstract

Introduction

Conclusions

References

Tables Figures

14

4

$\checkmark$

Back Close

Full Screen / Esc

Printer-friendly Version Interactive Discussion 


\section{Aspekte der Symbolik von Werbebildern und Stadträumen}

Um sich der Frage anzunähern, welche Inhalte und Bedeutungen raumbezogene Vorstellungsbilder transportieren, wurden Werbebilder aus Zeitschriften als methodisches Hilfsmittel verwendet. Bilder aus der Werbung schienen einerseits geeignet, um An5 regungen für Assoziationen mit dem jeweiligem Stadtteil zu liefern. Anderseits wurde auf diese Weise versucht, sich der Komplexität eines nicht exakt abgrenzbaren und benennbaren Lebensstils anzunähern, der - so die Hypothese - auf einzelne Stadträume projiziert wird. Da ein wesentlicher Teil der Werbebotschaften auf einer unbewussten Ebene kommuniziert wird und Gefühle, Wertungen und Sehnsüchte nur in bildhaften

10 Symbolen erkennbar sind, sollte das methodische Verfahren der Bildassoziation auch einen Zugang zu den latenten kollektiven Deutungsmustern von Quartieren eröffnen. Ausgangspunkt war zum einen die Überlegung, dass die in der Gesellschaft tatsächlich vorhandenen gesellschaftlichen Strukturen, Verhältnisse und Stiltypen von den bilderfixierten Massenmedien aufgegriffen und weitergereicht werden (Eickelpasch, 1998:9f.).

15 Zum anderen die Annahme, dass die zum Zweck der Produktwerbung symbolisch dargestellten gesellschaftlichen Bedürfnisse und Lebensstile prinzipiell auch geeignet sind, sich einer subjektiv erlebten Situation, Lebensweise oder Atmosphäre in einem Wohngebiet anzunähern. Werbung als Sinn- und Identitätsstifter postmoderner Gesellschaften kann dabei als Seismograph des Zeitgeists gelten. Die narrative Struktur eines Werbebildes entspricht einer Folie, die grundlegende menschliche Bedürfnisse wie Sicherheit, Selbstbestätigung, Macht und Gemeinschaft abbildet (Führer, 2003:146). Die überwiegend visuellen Botschaften menschlicher Bedürfnisse und gesellschaftlich relevanter Mythen in der Werbung, lassen sich auf andere Bereiche wie den Raum übertragen, der in ähnlicher Weise die Funktionen eines Labels für gesellschaftliche Werte und identitätsstiftende Kategorien (z.B. Sozialstatus, Herkunft) übernehmen kann (Shields, 2004:2). Zu diesem Zweck wurden Werbeanzeigen in den
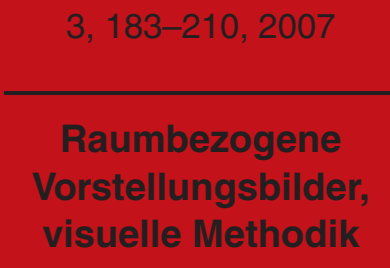

Title Page

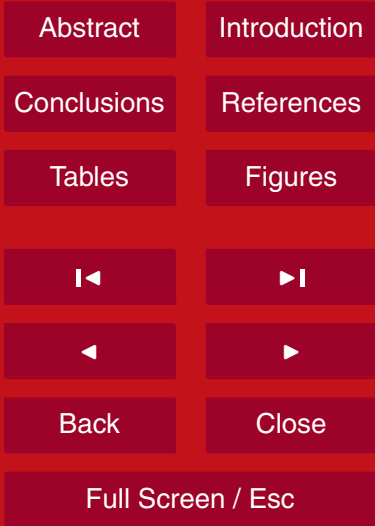

Printer-friendly Version Interactive Discussion 
gängigen deutschen Illustrierten ${ }^{2}$ über einen Zeitraum von drei Wochen gesichtet, wobei anzumerken ist, dass viele Werbekampagnen parallel in verschiedenen Magazinen zu finden sind. Einzige Vorgabe war zunächst die Suche nach Werbebildern, auf denen eine realitätsnahe Situation erkennbar ist, von der vorstellbar ist, dass sie tatsächlich 5 irgendwo in einer europäischen Stadt fotografiert wurde. Mit dieser Vorgabe reduzierte sich die Zahl der möglichen Bilder bereits ganz erheblich, da die meisten Werbekampagnen mit abstrakten, surrealen oder exotischen Darstellungen arbeiten. Um die Bilderzahl in der Befragungssituation überschaubar zu halten, wurde die Auswahl, die aus einen Pool von 12 Bildern bestand, auf neun Fotos beschränkt (s. Abb. 1a bis i). Die 10 Symbolkonfigurationen der Werbebilder stützen sich u.a. auf die Elemente: Alter und Geschlecht der dargestellten Personen, Einrichtungsgegenstände (wie Spiegel, Sofa), Kleidung und Schmuck, sonstige Gegenstände wie Fahrrad, Auto, Rosen, Musikinstrumente sowie Hinweise auf soziale Zusammenhänge (z.B. Familie Bild B, Freunde Bild C, I). Diese Konfigurationen legen dem Betrachter in einem spezifischen sozialen Kontext ein bestimmtes Bedeutungsschema als zutreffende Interpretation nahe und lassen weitere idealtypische Personenmerkmale mitassoziieren (Hölscher, 1998:76). Grundsätzlich war es ursprünglich bei diesem empirischen Experiment aber nicht das Ziel, die in der Gesellschaft vorhandenen Lebensstile idealtypisch oder vollständig abzubilden. Intention war es zunächst lediglich einen provokanten Input für Gespräche über raumbezogene Vorstellungen bereit zu stellen. Das hochgradig stereotypisierte und selektiv produzierte Werbematerial, das aber gleichzeitig assoziativ wirkt und interpretativ offen ist, schien dafür geeignet.

Die Bilder wurden sowohl in repräsentativen Haushaltsbefragungen vorgelegt ${ }^{3}$, als auch in den Tiefeninterviews mit Gebietsbewohnern und Immobilienmaklern. Insge-

\footnotetext{
${ }^{2}$ Spiegel, Stern, Bunte, Gala, Frau im Spiegel, Focus, Brigitte, Für Sie, verschiedene Stadtmagazine.

${ }^{3}$ Mit einer Stichprobengröße von $\mathrm{N}=298$ im Waldstraßenviertel, $\mathrm{N}=320$ in der Südvorstadt und $\mathrm{N}=230$ am Neustädter Markt wurden im Jahr 2002 im Mittel 9\% aller Haushalte in den Untersuchungsgebieten befragt.
}

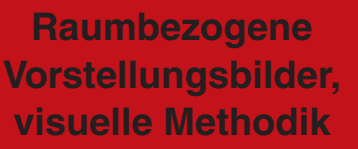

Title Page 
samt wurden 22 Intensivinterviews mit Personen geführt, die seit Mitte der 1990 Jahre in die Untersuchungsräume zugezogen sind, sowie neun Gespräche mit ortsansässigen Vertretern der Immobilienbranche. Wurde in den repräsentativen Haushaltsbefragungen nur gefragt welches Bild zum eigenen Wohngebiet passt und Möglichkeiten 5 zur offenen Assoziationen gegeben, wurden die Bilder in den Interviews sowohl in ihren möglichen Bezügen auf das Stadtviertel als auch auf den eigenen Lebensstil intensiver diskutiert. Qualitative und quantitative Verfahren haben sich hier ergänzt und gezeigt, dass zum einen die Werbebilder relativ übereinstimmend decodiert werden, und zum anderen auch auf bestimmte räumliche Umgebungen übertragen werden 10 können. So waren die Befragten fast alle in der Lage, einen Teil der Bilder mit der subjektiv wahrgenommenen Situation in ihrem Wohnviertel in Verbindung zu bringen. ${ }^{4}$ Die starke Übereinstimmung unter den Befragten bei den schriftlich festgehaltenen Bildassoziationen zeigt dies besonders deutlich und bestätigt die Annahme, dass ein gesellschaftlicher Grundkonsens in der Einordnung der dargestellten Situationen vorliegt. Gleichzeitig zeigen sich signifikante Unterschiede in der Auswahl der Bilder, die den drei Gebieten jeweils zugeordnet wurden. Neben ambivalenten Bewertungen, die vor dem Hintergrund individueller Projektionen und biographischer Erfahrungen zu verstehen sind, wurden überindividuelle in das Alltagswissen eingewobene, gesellschaftliche Übereinkünfte über den jeweiligen Status der Quartiere und über deren Platzierung im kulturellen System bis hin zu Stereotypen deutlich. Sie verweisen damit auf das Vorhandensein raumbezogener, sozialer Repräsentationen im Sinn von wertenden Vorstellungen, die von den Gesellschaftsmitgliedern weitgehend geteilt werden. Nachfolgende Ausführungen sollen dies beispielhaft veranschaulichen. Dabei wird auch auf die Symbolsprache der einzelnen Werbebilder genauer eingegangen.

\footnotetext{
${ }^{4}$ Die Verweigerungsquote dieser Frage lag zwischen $7 \%$ im Waldstraßenviertel und $11 \%$ am Neustädter Markt.
}

Raumbezogene Vorstellungsbilder visuelle Methodik

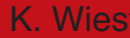

Title Page

Abstract Introduction

Conclusions References

Tables $\quad$ Figures

I

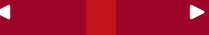

4

$\triangleright$

Back

Close

Full Screen / Esc

Printer-friendly Version

Interactive Discussion 


\section{Werbebilder und Raumbilder als Labels gesellschaftlicher Wertvorstellungen}

\subsection{Urbanität und Nonkonformismus - die Südvorstadt als Vorstellungsraum}

In der Südvorstadt bezogen sich die Gesprächspartner im Allgemeinen auf den Mythos des freien und toleranten Stadtlebens. Lebendigkeit, Trubel sowie die Erfahrbarkeit von Unterschiedlichkeit wurden immer wieder zur Charakterisierung dieses Quartiers herangezogen. Damit werden auf die Südvorstadt Vorstellungsinhalte übertragen, die dem Bild vom urbanen Leben sehr nahe kommen, das dem Leitbild der alten, europäischen Stadt zugrunde liegt (Schroer, 2005:329). Dies spiegeln auch die Assoziationen mit den Bildern aus der Werbung wider, die für die Südvorstadt als passend ausgewählt 10 wurden. So hat sich Bild I zwar für alle drei untersuchten citynahen Gründerzeitgebiete als besonders treffend erwiesen (s. Abb. 2). Für die Südvorstadt erreicht diese Abbildung jedoch eine absolut herausragende Zahl an Nennungen: Dargestellt sind hier zwei junge Mädchen, die häufig als Studentinnen interpretiert wurden und die zusammen auf einem Fahrrad sitzen. Diese Abbildung stellt offensichtlich eine Situation dar, die das Lebensgefühl des Stadtquartiers in besonderer Weise zu treffen scheint. Sie wurde von den Befragten im offenen Assoziationsverfahren spontan mit den Begriffen „jung“ „lebendig“ „unkonventionell“ in Verbindung gebracht wird. Dabei wurde das Bild vielfach in einem positiven Sinn, als frisches Infragestellen bzw. Überschreiten gesellschaftlicher Normen gelesen. Ausschlaggebend für diese Interpretation ist die junge

20 Frau, die auf dem Gepäckträger mitfährt - ein Verhalten, das nicht den Verkehrsregeln entspricht. Das Fahrrad wird in diesem Kontext zum Symbol für eine junge, alternative, unkonventionelle Lebensweise. Folgendes Zitat einer Bewohnerin gibt diese Lesart des Werbefotos wieder: als eine Interpretationsfolie, die sich auf die Südvorstadt übertragen lässt und ebenso für den Betrachter Identifikationspotenziale bietet:

25 „Jung, lebensfroh, wahrscheinlich Studenten, Studentinnen. Ja, das Fahrrad natürlich, als wichtigstes Element der Südvorstadt. . [...]..das hat so was Ungezwungenes. Noch nicht erwachsen sein. Lebensfreude. Nicht konform. Man sitzt ja nicht auf dem Gepäckträger, das darf man nicht, aber ich würde es genauso machen" (Museologin, 33 Jah-

Title Page 
re).

Neben Bild C, das in der Südvorstadt häufig mit der dichten Kultur- und Kneipenszene und einer alternativen bzw. gemeinschaftsorientierten Lebensweise in Verbindung gebracht wurde, wurde Bild $A$ an dritter Stelle, häufiger als in den anderen beiden 5 Untersuchungsgebieten als passend ausgewählt. Der Darstellung einer rothaarigen Frau auf einem Sofa wurde zu 45\% die Ausstrahlung von „Unabhängigkeit“ „Coolness/Flippigkeit“ und „Kreativität" zugesprochen. Dabei wird eine Überlagerung von individuellen Projektionen und kollektiven Deutungsmustern auch darin erkennbar, dass Bild A überdurchschnittlich häufig von jüngeren, besser verdienenden Singles als zum 10 Quartier passend ausgewählt wurde: Haushalte, die dem Typ des Gentrifiers zugeordnet werden könnten, scheinen Darstellung A in ihrem Wohngebiet häufiger auszumachen. Dabei enthält das Bild Wiedererkennungswerte, die zum Teil eher mit sozialen Repräsentationen Berlins in Zusammenhang gebracht werden: „Bild A, die Junge, Hippe, Erfolgreiche passt nicht ganz [zur Südvorstadt d.V.]. Das ist irgendwie ein Haupt-

15 stadtbild. Sehr kühl, sehr trendy mit der roten Perücke und dem coolen Hintergrund. Also [...] Leipzig ist zu gemütlich für dieses Bild“" (Studentin, 25 Jahre).

Durchgängig für das Vorstellungsbild „Südvorstadt“ erscheint aber eine unkonventionelle Atmosphäre, der entspricht, dass die Bilder, die als am wenigsten zur Südvorstadt passend erschienen, Hinweise auf eine konventionelle Lebensweise enthalten: Diese wird z.B. über das Bild der Kleinfamilie aus der Ramawerbung (B) oder den Anzugträger in Bild G vermittelt. Die Ablehnung dieser Bilder für die Südvorstadt steht teilweise im Kontrast zu deren Beurteilung in Bezug auf das Waldstraßengebiet.

4.2 Wohlstand, Erfolg und Harmonie - das Waldstrassenviertel als Vorstellungsraum

Die Zuordnung der Werbebilder fiel im Waldstraßengebiet weniger eindeutig aus als in 25 der Südvorstadt. Hier konzentrierten sich die Nennungen auf fünf Bilder, die von den Befragten mit den Begriffen „Kinder- bzw. familienfreundlich“ (Bild B und F), „Gründerzeitflair" (Bild F und I), „Nostalgie“ (Bild F und C) sowie „Stil“" „Noblesse“ (Bild H und F) in Verbindung gebracht wurden. Insbesondere die sprichwörtlich gewordene „Ra- 
mafamilie“ (Bild B), die den modernen Mythos der idyllischen Kleinfamilie und des harmonischen Familienglücks anspricht, wurde von vielen Befragten im Waldstraßenviertel verortet. Obstschale und Frühstücksituation im Freien als Zeichen für Gesundheit, Natürlichkeit wurden mit einer, in diesem Wohngebiet erlebten, „heilen Welt“ bzw. Ge5 borgenheit in Verbindung gebracht:

„... es soll eben die glückliche, sonnige Familie sein, mit vitaminreichem Frühstück, und das ist hier so auch der Fall. Meine Empfindung ist hier auch, dass Familien, die hier wohnen, häufig sehr anspruchsvolle, bewusste Menschen sind, die wissen, wie sie sich kleiden, was sie essen und wie sie mit ihren Kindern den Sonntagmorgen 10 beginne." (freiberuflicher Architekt, 35 Jahre).

Es sind aber vor allem die „Wohlstandskinder“ bzw. die ,jungen Mittelstandsfamilien" die im Kontext von Werbebildern und Quartier thematisiert werden. So wurde das in Bild F dargestellte Mädchen mit Hut häufig als Chiffre für eine „behütete Kindheit" gelesen. Entsprechende Lebensbedingungen werden nur an ganz bestimmten 15 Stellen der Stadt verortet. Folgende Interviewpassage verdeutlicht diese unmittelbare Verknüpfung von sozialen Verhältnissen mit bestimmten Stadträumen: „Aber das Kind sieht schon nach Wohlstandskind aus, wie es sich da mit dem Kleid und dem großen Hut von seiner Mutti verkleidet. Von daher würde ich am ehesten sagen: Gohlis, Waldstraßenviertel" (Studentin 25 Jahre). Gleichzeitig vermitteln die verspielte Kleidung, antike Einrichtungsgegenstände und das in Sepiafarben gehaltene Foto den Eindruck einer nostalgischen Darstellung aus vergangenen Kindertagen bzw. der "guten alten Zeit‘'Der Bezug auf vergangene Zeiten klingt auch in Assoziationen mit Bild C an: das in Schwarz-Weiß gehaltene Foto mit einer Gruppe von Menschen mit Musikinstrumenten wurde von 39\% der Befragten mit Tradition und Geselligkeit in Verbindung gebracht.

25 Dabei wurde von einigen Gesprächspartnern eine direkte Verbindung zur jüdischen Geschichte des Quartiers hergestellt und mit den Musikern eine Klezmerkapelle assoziiert. Die in den Bildern $\mathrm{F}$ und $\mathrm{C}$ dargestellten nostalgischen Lebensweisen erscheinen als Gegenentwürfe zu Zweckrationalität und Modernität. Unter dem Aspekt ihrer Zuordnung zu bestimmten Stadträumen erscheint der Verweis auf Studien von Interesse, die

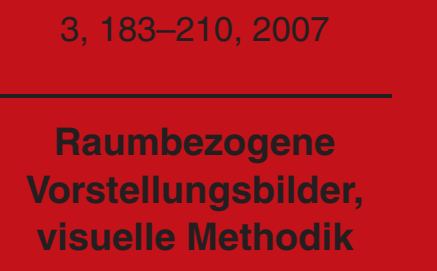

\section{Title Page}

Abstract

Introduction

Conclusions

References

Tables Figures

14

4

Back Close

Full Screen / Esc

Printer-friendly Version Interactive Discussion 
die Kombination von Geschichtlichkeit und Moderne als entscheidenden Bestandteil der urbanen Gentrificationästhetik identifizieren (Phillips, 2002:292). Bezeichnend erscheint in diesem Zusammenhang, dass die Bilder F, C und A überdurchschnittlich häufig von Bewohnern des Waldstraßengebiets als passend ausgewählt wurden, die 5 man dem Typ des Gentrifiers ${ }^{5}$ zuordnen könnte.

Auch Werbefoto $\mathrm{H}$ konnte relativ häufig mit dem Waldstraßengebiet in Verbindung gebracht werden. Es wurde allerdings von den Befragten oft als ein Bild angesehen, das eher dem Image als der Realität des Wohngebiets entspricht. Indem der Bildbetrachter scheinbar vom Inneren einer (italienischen) $\mathrm{Bar}^{6}$ auf den Protagonisten der 10 Szene blickt, entsteht der Eindruck, dass auf diesen Mann gewartet wird. Dass es sich um einen Menschen handelt, der auf der erfolgreichen Seite des Lebens steht (sowohl im Beruf als auch bei den Frauen), können der Rosenstrauß, die Kleidung und das Lächeln andeuten. Insgesamt symbolisieren die einzelnen Bildelemente und nicht zuletzt das beworbene Auto selbst Wohlstand, Erfolg und Überlegenheit - soziale Ka15 tegorien, die auch auf das Quartier übertragen wurden: „... und Bild $H$ ist eigentlich fast das typischste. Also wie so ein Prototyp, würd ich fast sagen. Ein Riesenstrauß roter Rosen, ein Mann in den besten Jahren im schwarzen Anzug mit nem Auto dahinter. Also, das könnte hier irgendwo in jeder Straße vom Waldstraßenviertel sein. Der würde nicht auffallen als was Besonderes. ...jung, dynamischer Manager, erfolgsreich, oder so was, der zu seiner Freundin geht... "( Filialleiterin, 44 Jahre).

Die Bildauswahl zeigt, dass die untersuchten Gebiete für die Bewohner ganz unterschiedliche, zum Teil kontrastierende Lebenswelten repräsentieren. So wurden die Bilder, die nach Ansicht der Befragten besonders wenig zum Waldstraßenviertel passen (D und $E$ ), am häufigsten für den Neustädter Markt ausgewählt.

\footnotetext{
${ }^{5}$ Beruflich etablierte Bewohner mit überdurchschnittlichen Bildungsgrad und Einkommen.

${ }^{6}$ die Buchstaben AR sind spiegelverkehrt zu erkennen
}

\section{SGD}
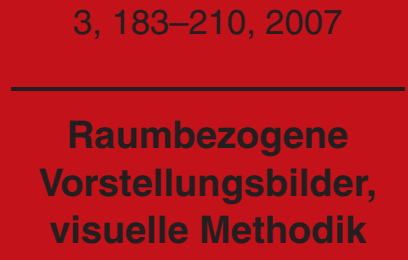

Title Page

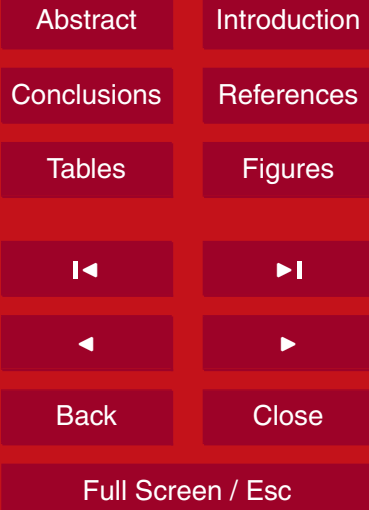

Printer-friendly Version Interactive Discussion 
In den Gesprächen mit den Bewohnern des Neustädter Marktes wurden häufig gesellschaftliche Probleme die mit sozialer Ausgrenzung verbunden sind, wie Arbeitslosigkeit, Drogenmissbrauch und Kriminalität angesprochen. Diese Themen finden sich

5 z.T. auch wieder in den für den Stadtteil am häufigsten als passend ausgewählten Werbefotos. So wurden die in Bild E erkennbaren Attribute weißes Unterhemd, Pantoffeln, aber auch stark blondierte Haare durchgängig als Symbolkonfiguration einer proletarischen Lebensweise gelesen, die häufig unmittelbar mit sozialen Problemen gleichgesetzt wurde. Die in dieser Darstellung ironisierend verwendeten Symbole und Zeichen 10 weisen so eine typische Konfiguration auf, die einer bestimmten Schematisierung der Sozialwelt entspricht und mit ihr spielt (Hölscher, 1998: 66). So assoziierten 49\% der Befragten mit diesem Bild Sozialfälle bzw. Arbeitslosigkeit und 12\% Alkoholprobleme, obwohl auf dem Bild keine Alkoholika erkennbar sind. Wie im folgenden Interviewzitat wurde diese raumbezogene Vorstellung zwar in vielen Fällen von den Gesprächspart15 nern als ein ironisches Konstrukt erkannt - gleichzeitig kommt in der Wahl des Bildes der Rückgriff auf ein intersubjektiv geteiltes Wissen über diesen Stadtteil zum Ausdruck: „Ja natürlich. Bild E. Passt natürlich klischeemäßig total. Das ist ja das, was man sich hier so vorstellt. ... weil man ja auch weiß und weil man sie ja auch sieht. Also der typische Sozialhilfeempfänger wie man ihn sich zu Hause vorstellt. Gut, so 20 in Trainingshose und Hemd, wie er auch zu Aldi geht. Ja. Passt schon“ (Student, 26 Jahre).

Neben Bild I wurde auch Werbefoto D als charakteristisch für den Leipziger Osten angesehen. Im Kontext dieses Bildes werden schwerer Schmuck auf dunkler Kleidung und Kopftuch als Zeichen benutzt, um Assoziationen mit der Hiphop-Szene hervorzurufen. Die düsteren Farben und der Gesichtsausdruck dieses „,Gangsta-Rappers“ wecken dabei Erinnerungen an das archetypische Bild des outlaws. Dabei assoziierten die Befragten mit diesem Bild häufig Ausländer (46\%) sowie Kriminalität und Drogen (11\%). Diese Stereotype wurden gleichzeitig überdurchschnittlich häufig auf den

Raumbezogene Vorstellungsbilder, visuelle Methodik

Title Page

Abstract Introduction

Conclusions References

Tables Figures

$1<$

4

$>$

Back

Full Screen / Esc

Printer-friendly Version

Interactive Discussion 
Neustädter Markt übertragen. Die Ausstrahlung von Verschlossenheit und „sich trotzig durchs Leben kämpfen und beißen müssen" der dargestellten Person erwies sich als eine Ursache dafür, dass dieses Bild wesentlich seltener in der Südvorstadt angesiedelt wurde. Das Aussehen wurde damit mit einer sozialen Lebenslage gleichgesetzt: 5 „Ja, dieser Blick, der so unter Druck setzt, den findet man auch dort, wo Leute eben wenig Geld haben. Das ist ganz klar" (Student, 24 Jahre). Folgt man den Ergebnissen der Bild-Assoziationen und ihrer räumlichen Zuordnung zu bestimmten Stadtteilen, scheint der Leipziger Osten eher als Ort erlebt zu werden, an dem sich die unfreiwillig oder freiwillig am Rande der Gesellschaft stehenden befinden und der sich auch 10 kulturell deutlich vom Rest der Stadt unterscheidet. Wie im folgenden Zitat wird dieser Stadtteil dabei als kulturell eigenständige Einheit wahrgenommen: „.. der Leipziger Osten ist jetzt schon ein bisschen eine andere Welt. Und wenn dann die Migranten einziehen, dann wird es umso mehr eine andere Welt. . " (Angestellter, 32 Jahre). Dabei wurde gerade in der Gegenüberstellung mit den anderen beiden Untersuchungs15 quartieren ein System symbolischer Positionen bzw. Ordnung erkennbar, durch das der einzelne Stadtraum in Relation zu anderen gesetzt wird und in diesem Sinn die Aussage bestätigt, „that "place image" is not constructed in a purely local manner but with an eye to other places..." (Shields, 2004:5). Die Ergebnisse der Bildassoziationen können dies verdeutlichen durch die konträre Bewertung der Stadträume: Ebenso wenig wie die für den Neustädter Markt als charakteristisch empfundenen Bilder für das Waldstraßenviertel ausgewählt wurden, wurden die im Waldstraßengebiet als besonders passend empfundenen Bilder B und $\mathrm{H}$ für den Neustädter Markt gewählt. Bezogen auf das in B dargestellte Familienfrühstück formulierte dies eine Studentin folgendermaßen: „... Bild B passt überhaupt nicht, weil diese Leute einfach zu sehr in einer 25 heilen Welt wohnen, und der Neustädter Markt momentan diese Welt nicht repräsentiert" (Studentin, 27 Jahre). Diese wie auch andere Aussagen verdeutlichen, dass innerhalb der informellen Strukturen des Alltags Orte unterschiedliche gesellschaftliche Klischees transportieren, die sowohl private als auch öffentliche Entscheidungen steuern und beeinflussen.

\section{SGD}

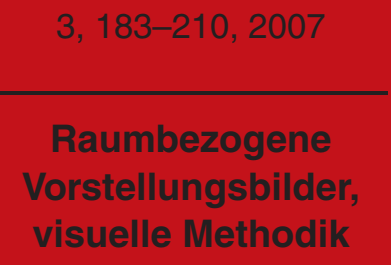

Title Page

Abstract Introduction

Conclusions References

Tables $\quad$ Figures

1

4

$\checkmark$

Back

Full Screen / Esc

Printer-friendly Version

Interactive Discussion 


\section{Raumbezogene Repräsentationen zum Zweck der Vermarktung}

In Ergänzung zu Raumvorstellungen die im Alltag präsent sind, sollten Repräsentationen des Raumes, die in fachlichen Diskursen kommuniziert werden, beleuchtet werden. Auf der Grundlage von Informations- und Werbematerialien aus den Bereichen 5 Immobilienwirtschaft, Stadtmarketing und Standortwerbung die zwischen Juli und August 2004 öffentlich zugänglich waren, wurde der Frage nachgegangen, mit welchen Attributen und Symbolwerten die drei Leipziger Untersuchungsgebiete zum Zweck der Vermarktung belegt werden. Voraussetzung für die Auswahl des Materials war die Nennung des Ortsnamens in einem qualifizierenden Textzusammenhang. Die Recherche 10 von Druckerzeugnissen und Internetseiten, in denen die Namen der Untersuchungsquartiere als Werbung für den Standort, im Rahmen der Präsentationen lokaler Unternehmen oder zum Zweck der Vermarktung von Immobilien eingesetzt wurden, war jedoch nur bedingt erfolgreich. So erwiesen sich nur etwa $5 \%$ der Onlineangebote aus dem Bereich der Immobilienbranche für die Fragestellung als brauchbar. Die Analyse 15 des Informations- und Werbematerials wurde durch neun Intensivinterviews mit ortsansässigen Immobilienmaklern ergänzt. Als Ursache für die relativ geringe Bedeutung einer genaueren Darstellung des Immobilienstandorts in Anzeigen gaben die Makler die meist vorhandenen Ortskenntnisse der Wohnungssuchenden an, die genauere Quartiersbeschreibungen und den damit verbundenen Aufwand nicht rechtfertigen würden. Raumbilder scheinen im vorliegenden Fall demnach weniger von fachlichen Diskursen und Standortmarketing geprägt zu werden. Vielmehr gibt die Alltagspraxis den fachlichen Diskurs vor, indem auf der Basis der bereits bestehenden Alltagsbedeutungen neue Bedeutungen geschaffen und Räumen gezielt semantische Merkmale zugeordnet werden. Die in Exposes durch Texte und Bilder dargestellten Repräsenta25 tionen der Stadträume greifen bestehende Bedeutungen, die in den Codes der Altagskultur vorliegen auf und verbinden sie mit den Quartieren. Die Stadträume werden auf diese Weise als Entitäten behandelt, denen bestimmte Eigenschaften zugeschrieben werden. Deutlich wurde bei der Materialsuche allerdings, dass nicht alle drei Untersu-

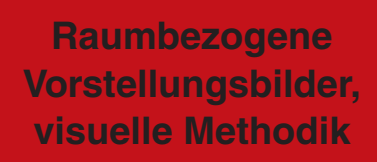

Title Page

Abstract

Introduction

Conclusions

References

Tables

Figures

$1<$

4

$\checkmark$

Back Close

Full Screen / Esc

Printer-friendly Version

Interactive Discussion 
chungsgebiete in gleichem Maß Gegenstand der Präsentationen von Stadtmarketing, Standortwerbung und Immobilienwirtschaft sind: Besonders schwierig gestaltete sich die Suche im Bereich Immobilienvermarktung für den Stadtraum Neustädter Markt. Oft wurden hier nur sehr knappe und relativ allgemein gehaltene Aussagen zu Aspek5 ten des Wohnumfelds formuliert, die sich vor allem auf die verkehrsgünstige Lage des Stadtteils bezogen. Dagegen lag vergleichsweise viel Informationsmaterial über Stadtteilprojekte und Quartiersmanagement vor. Mit Wendungen wie „vieles kann sich noch entwickeln“ „kommt in Fahrt“ „ist auf dem Weg“ wird in den Texten metaphorisch vermittelt, dass sich das Quartier in einem Prozess befindet, in dem der Anschluss an ein 10 bestimmtes Niveau erst noch erreicht werden muss. Dem entspricht auch das im Rahmen des Programms Soziale Stadt entwickelte Logo mit einem Sonnen-Smiliegesicht und nach oben weisendem Schriftzug, das vor allem die Aussicht auf eine positive Zukunft als zu erreichendem Ziel aufzeigt. Es soll Identifikationsangebote schaffen und steht für „den Willen, den Leipziger Osten trotz seiner Probleme zu einem un15 verwechselbaren, konkurrenz- und zukunftsfähigen Stadtteil Leipzigs weiter zu entwickeln“ (http://www.leipziger-osten.de).

Bei Wohnangeboten in den Untersuchungsgebieten Waldstraßenviertel und Südvorstadt wirbt demgegenüber die Immobilienbranche deutlich intensiver und spezieller mit der Lage. Dabei wird der Standort Waldstraßenviertel sowohl in Texten als auch 20 Fotos vorrangig unter dem Aspekt seiner materiellen Ästhetik behandelt. In den teilweise sehr aufwändigen Exposés sind hin und wieder ausführliche kulturhistorische Ausführungen zur architektonisch wertvollen Bausubstanz zu finden. Diese sind auf ein entsprechendes Verständnis der Adressaten ausgerichtet. Gezeigt werden bauliche Details wie Treppenhäuser, Eingänge oder Luftbilder, die die Geschlossenheit der 25 gründerzeitlichen Bausubstanz zeigen. Dem entsprechen die Formulierungen der Exposés mit Attributen wie „herrschaftlich“, „stilvoll“ und „repräsentativ“. Diese Begriffe stehen für die Zugehörigkeit zu einer bevorzugten, herrschenden Klasse, die mit „Exklusivität" auch die Tatsache eines Vorbehaltenseins für eine kleine Elite beinhalten. Die verwendeten Superlative wie „bestes“, „Top“, „erstklassig“ „,schönstes“ verweisen

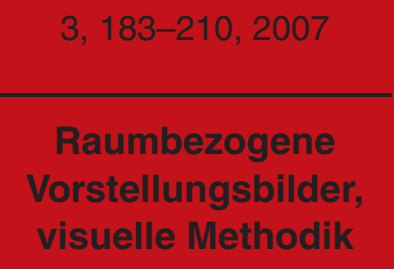

\section{Title Page}

Abstract

Introduction

Conclusions

Tables

References

\section{Figures}

$1<$

4

>

Back Close

Full Screen / Esc

Printer-friendly Version

Interactive Discussion 
auf eine bestehende Rangordnung, die eine kulturelle Überlegenheit impliziert und die durch den sprachlichen Mechanismus der Hierarchisierung soziale Identität und Differenz konstruieren (Hülsse, 2003:148).

Im Gegensatz zu den Darstellungen des Waldstraßenviertels, die Aspekte des Vier5 telslebens weitgehend ausblenden, wird die Südvorstadt häufig über Metaphern beschrieben, die dem Bildfeld eines ,,gesunden Organismus“ zuzuordnen sind. Wendungen wie „pulsierend“ „im Herzen“ „Iebendig“ „mitten im Leben“ „gesunde Mischung“ stehen für gesellschaftliche Vitalität und Zentralität. Diese Bilder entfalten besonders im Kontrast zum öffentlichen Diskurs um schrumpfende Städte ihre positive Wirkung, 10 der mit den Metaphern der Krankheit und des Absterbens verbunden ist.

Die Auswertung der Informationsbroschüren und Experteninterviews hat gezeigt, dass auch der fachliche Diskurs mit einer spezifischen Bildsprache verbunden ist. Diese bezieht sich im Leipziger Osten auf eine bessere Zukunft, im Waldstraßenviertel auf gesellschaftliche Hierarchien und Klassenzugehörigkeiten und in der Südvorstadt 15 auf Vitalität und Mittelpunktcharakter. Dabei muss angenommen werden, dass die im Kontext der Immobilienvermarktung verwendeten raumbezogenen Bilder nur einen geringen Einfluss auf das tatsächliche Wohnstandortverhalten der Bevölkerung haben. Dieser Einfluss kommt vermutlich eher bei ortsfremden, überregionalen Zuzügen zum Tragen und ist gleichzeitig schwer empirisch nach zu weisen. Bezogen auf sozialräum20 liche Differenzierungen zeigt die Auswertung jedoch vor allem, dass im Prozess gesellschaftlicher Kommunikation eine fortlaufende Verfestigung bestimmter Raumvorstellungen stattfindet. So werden die auf dem Alltagserleben basierenden Vorstellungen im Bereich der Vermarktung reproduziert und weitergereicht und wirken so wieder rückbestätigend auf die Stereotypisierung von Orten. Wird die realitätsvereinfachende
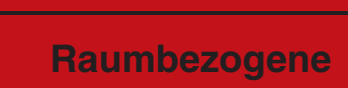

Vorstellungsbilder, visuelle Methodik

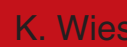

Title Page

Abstract Introduction

Conclusions References

Tables Figures

$1<$

4

$>$

Back

Close

Full Screen / Esc

Printer-friendly Version

Interactive Discussion 


\section{Fazit}

Die drei untersuchten Innenstadtquartiere sind Projektionsflächen für Schematisierungen der Sozialwelt, an die unterschiedliche, sich überlagernde Einstellungen, Gefühle und Lebensstile gekoppelt sind. Die Tatsache, dass das einzelne Untersuchungsgebiet 5 in deutlicher Opposition zu Räumen der Repräsentation steht, die mit anderen Werteund Kulturmustern verknüpft werden, verweist auf bereits verfestigte Vorstellungsinhalte bis hin zu stereotypen Bildern. Die Präsentationen der Quartiere in Werbe- und Informationsbroschüren beziehen sich auf diese kollektiven Bilder und könnten unter dem Aspekt der Vermarktung zu identitätsstiftenden Labels weiterentwickelt werden.

10 Am Beispiel des Leipziger Ostens wurde dieser Versuch deutlich, indem die kollektive Erfahrung eines sozial abgehängten und problembehafteten Stadtteils in das Bild einer besseren Zukunft übergeleitet werden soll.

Dabei handelt sich bei den Untersuchungsgebieten offensichtlich um Orte, an denen kulturelle Aushandlungsprozesse relativ intensiv stattfinden. Gleichzeitig ist auch 15 darauf hinzuweisen, dass in Leipzig über andere städtische Teilräume möglicherweise kaum ähnlich stark ausgeprägte Vorstellungen vorliegen. Als wesentliches und geeignetes Hilfsmittel um sich den Raumbildern in den Köpfen anzunähern, hat sich das verwendete Bildmaterial aus der Werbung erwiesen. Dabei wurde deutlich, dass Orte, ähnlich wie beworbene Produkte, Stellvertreterfunktionen für andere, nichtmaterielle, identitätsstiftende soziale Kategorien und Vorstellungen übernehmen können. Werden gesellschaftliche Schematisierungen und Stereotype im Alltag an bestimmte Räume gebunden, werden sie im Kontext der Werbung über bildhaft präsente Vorstellungsinhalte an Produkte gekoppelt. Vor dem Hintergrund der empirischen Ergebnisse erscheint es durchaus viel versprechend, diesen Versuch, Repräsentationen mit Hilfe visuell kommunikativer Werbebilder nachzuspüren, im Rahmen bildtheoretischer Erkenntnisse stärker zu vertiefen und weiterzuentwickeln. Das kulturelle Labeling von Orten weist dabei über informelle Altagsstrukturen hinaus, indem es auch politische und ökonomische Entscheidungen rahmen kann. Abschließend bleibt daher die Frage,

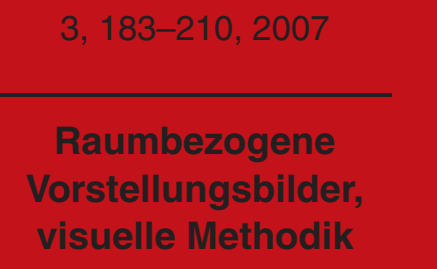

Title Page

Abstract

Introduction

Conclusions

References

Tables Figures

1

4

Back Close

Full Screen / Esc

Printer-friendly Version

Interactive Discussion 
welche Potenziale die Beschäftigung mit sozialen Verräumlichungen und „ThirdspaceGeographien“ für konkrete urbane Probleme bietet. Da davon ausgegangen werden kann, dass die Vorstellungsbilder über Stadträume Entwicklungen, die diesen sozialen Zuschreibungen entsprechen, erheblich befördert haben, sind sie ein wesentlicher Fak5 tor sozialräumlicher Differenzierung bis hin zur Segregation. Kulturelle Bewertungen von Orten sind aber durchaus im positiven wie negativen Sinn manipulierbar. Erkenntnisse über die Bilder in den Köpfen und die vom Zeitgeist abhängigen und kulturell begrenzten Deutungen von Räumen könnten so auch im Sinn gesellschaftlicher und urbaner Problemlösungen genutzt werden.

\section{Literatur}

Best, U. und Gebhard, D.: Ghetto - Diskurse. Geographie der Stigmatisierung in Marseille und Berlin, Praxis Kultur- und Sozialgeographie 24, Potsdam, 2001.

Caulfield, J.: Gentrification and desire. Canadian Review of Social Anthropology 26, 616-632, 1989.

15 Eickelpasch, R.: Struktur oder Kultur? Konzeptionelle Probleme der soziologischen Lebensstilanalyse, in: Verlust der Sicherheit? Lebensstile zwischen Multioptionalität und Knappheit, herausgegeben von: Hillebrandt, F., et al., 9-25, Westdeutscher Verlag, Opladen, Wiesbaden, 1998.

Führer, B.: Werbung als Vermittler mythischer Narration. Diplomarbeit an der Universität Wien. Fachbereich Publizistik und Kommunikationswissenschaft, Diplomica, Hamburg, 2003.

Hamnett, C.: The blind men and the elephant. the explanation of gentrification. Transactions, Institute of Britisch Geographers 16, 259-279, 1991.

Hill, A. und Wiest, K.: Sanfte Gentrifizierung, Studentifizierung und Inseln ethnischer Konzentration in ostdeutschen Innenstadtrandgebieten? Raumforschung und Raumordnung 6, 361374, 2004.

Hölscher, B.: Lebensstile durch Werbung? Zur Soziologie der Lifestyle-Werbung, Westdeutscher Verlag, Opladen, 1998.

Hülsse, R.: Metaphern der EU-Erweiterung als Konstruktionen europäischer Identität, Nomos, Baden-Baden, 2003.

Title Page

Abstract Introduction

Conclusions References

Tables $\quad$ Figures

14

DI

4

$>$

Back

Close

Full Screen / Esc

Printer-friendly Version

Interactive Discussion 
Lang, B.: Mythos Kreuzberg. Ethnographie eines Stadtteils 1961- 1995, Campus, Frankfurt a. M., 1998.

Lees, L.: Urban geography: discourse analysis and urban research, Progress in Human Geography 28, 1, 101-107, 2004.

5 Lees, L.: A reappraisal of gentrification: towards a "geography of gentrification", Progress in Human Geography 24, 3, 389-408, 2000.

Lefebvre, H.: La production de l'espace. Anthropos, Paris, 1974.

Ley, D.: The new middle class and the remaking of the central city, Oxford, 1996.

McCann, E. J.: Race, protest, and public space: Contextualizing Lefebvre in the U.S. City, Antipode, 163-184, 1999.

Miggelbrink, J.: Der gezähmte Blick. Zum Wandel des Diskurses über Raum und Region in humangeographischen Forschungsansätzen des ausgehenden 20. Jahrhunderts, Beiträge zur regionalen Geographie 55, Leipzig, 2002.

Mills, C.: Myths and meanings of Gentrification, in: Place/Culture/representation, herausgege15 ben von: Duncan J. a. D. Ley, 149-170, Routledge, London, 1993.

Phillips, M.: The production, symbolisation and socialisation of gentrification: impressions from two Berkshire villages, Transactions of the Institute of British Geographers, 282-308, 2002.

Schelske, A.: Bedeutung oder Bezeichnung in: Vom Realismus der Bilder: Interdisziplinäre Forschung zur Semantik bildlicher Darstellungsforme, herausgegeben von: Sachs-Hombach, K.

20 u. K. Rehkämper, 147-158, Magdeburg, 2001

Schmid, Ch.: Stadt, Raum und Gesellschaft. Zur Theorie der Produktion des urbanen Raumes von Henri Lefebvre, Sozialgeographische Bibliothek 1, Steiner Verlag, München, 2005.

Schroer, M.: Stadt als Prozess. Zur Diskussion städtischer Leitbilder. In: Die Wirklichkeit der Städte, Soziale Welt 16, herausgegeben von: Berking, H. u. M. Löw, 327-344, Nomos, Baden-Baden, 2005.

Shields, R.: Places on the margin, Routledge, London, 1991.

Shields, R.: Intersections in Cultural Policy: Geographical, Socioeconomic and Other Markers of Identity. (http://www.spaceandculture.org/robshields/papers/ intersectionsculturalpolicy-rshields.pdf) 2004.

so Soja, E. W.: Thirdspace. Journeys to Los Angeles and Other Real- and-Imagined Places. Blackwell Publishers, Cambridge, Massachusetts, 1996.

Wiest, K. und Zischner, R.: Aufwertung innerstädtischer Altbaugebiete in den neuen Bundesländern - Prozesse und Entwicklungspfade, Deutsche Zeitschrift für Kommunalwissen- 
schaften I, 99-121, 2006.

http://www.leipziger-osten.de (Datum: 31.05.2006).

SGD

3, 183-210, 2007

Raumbezogene Vorstellungsbilder, visuelle Methodik

K. Wiest

Title Page

Abstract Introduction

Conclusions References

Tables

Figures

14

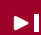

4

Back

Close

Full Screen / Esc

Printer-friendly Version

Interactive Discussion 
Tabelle 1. Sozialräumliche Differenzierung als gesellschaftlicher Produktionsprozess. Auf der Grundlage von Lefebvre (1974); McCann (1999:172); Miggelbrink (2002:51); Phillips (2002); Schmid (2005:210-226); Soja (1996).

\begin{tabular}{|c|c|c|}
\hline $\begin{array}{l}\text { Theoretische Raum- } \\
\text { konzeptionen }\end{array}$ & & $\begin{array}{l}\text { Gesellschaftliche Produktion } \\
\text { gentrifizierter und margnialisierter } \\
\text { Räume }\end{array}$ \\
\hline $\begin{array}{l}\text { Firstspace - das } \\
\text { Wahrgenommene } \\
\text { räumliche Praxis }\end{array}$ & $\begin{array}{l}\text { Eigentumserwerb, } \\
\text { Investitionen in den Baubestand durch Sanierung etc., } \\
\text { „Invasion“ und „,Kolonisierung“ durch Neuzuzug } \\
\text { Verdrängungsprozesse }\end{array}$ & $\begin{array}{l}\text { Die Produktion von materiellen } \\
\text { Gegebenheiten } \\
\text { Materielle Artefakte, } \\
\text { bauliche Gegebenheiten, } \\
\text { Infrastruktur, } \\
\text { soziale Strukturen }\end{array}$ \\
\hline $\begin{array}{l}\text { Secondspace - das } \\
\text { Konzipierte } \\
\text { Repräsentation des } \\
\text { Raumes }\end{array}$ & $\begin{array}{l}\text { Ausweisung von Fördergebieten, } \\
\text { z.B. Denkmalschutz-, Sanierungsgebiete, Programmge- } \\
\text { biete „Soziale Stadt“ } \\
\text { Städtebauliche Leitbilder wie z.B. „Europäische Stadt“ } \\
\text { „Revitalisierung“ } \\
\text { Präsentation von Stadträumen zum Zweck der Vermark- } \\
\text { tung, branding, } \\
\text { Immobilienbewertung } \\
\text { Texte in Lifestylemagazinen und Reiseführern }\end{array}$ & $\begin{array}{l}\text { Die Produktion von Wissen: } \\
\text { Diskurse in Planungspraxis, Wis- } \\
\text { senschaft, Medien, Werbung }\end{array}$ \\
\hline $\begin{array}{l}\text { Thirdspace - } \\
\text { das Gelebte } \\
\text { Räume der Re- } \\
\text { präsentation, } \\
\text { Vorstellungsräume }\end{array}$ & $\begin{array}{l}\text { Identifikation durch Symbole und Codes, } \\
\text { Symbolische Barrieren durch die Projektion von sozialen } \\
\text { Zuschreibungen, } \\
\text { Wohnadresse als symbolisches Kapital } \\
\text { Inszenierung städtischer Räume (Architektur, Design, } \\
\text { Ikonographie), } \\
\text { Kulisse für Film, Werbung, Literatur }\end{array}$ & $\begin{array}{l}\text { Die Produktion von Bedeutungen } \\
\text { Raumbezogene Bilder und Sym- } \\
\text { boliken z.B. } \\
\text { "Szene-Viertel" "Ghetto“, Mythos } \\
\text { von Orten }\end{array}$ \\
\hline
\end{tabular}

SGD

3, 183-210, 2007

Raumbezogene

Vorstellungsbilder,

visuelle Methodik

K. Wiest

Title Page

Abstract Introduction

Conclusions References

Tables Figures

IS

4

Back

Close

Full Screen / Esc

Printer-friendly Version

Interactive Discussion 


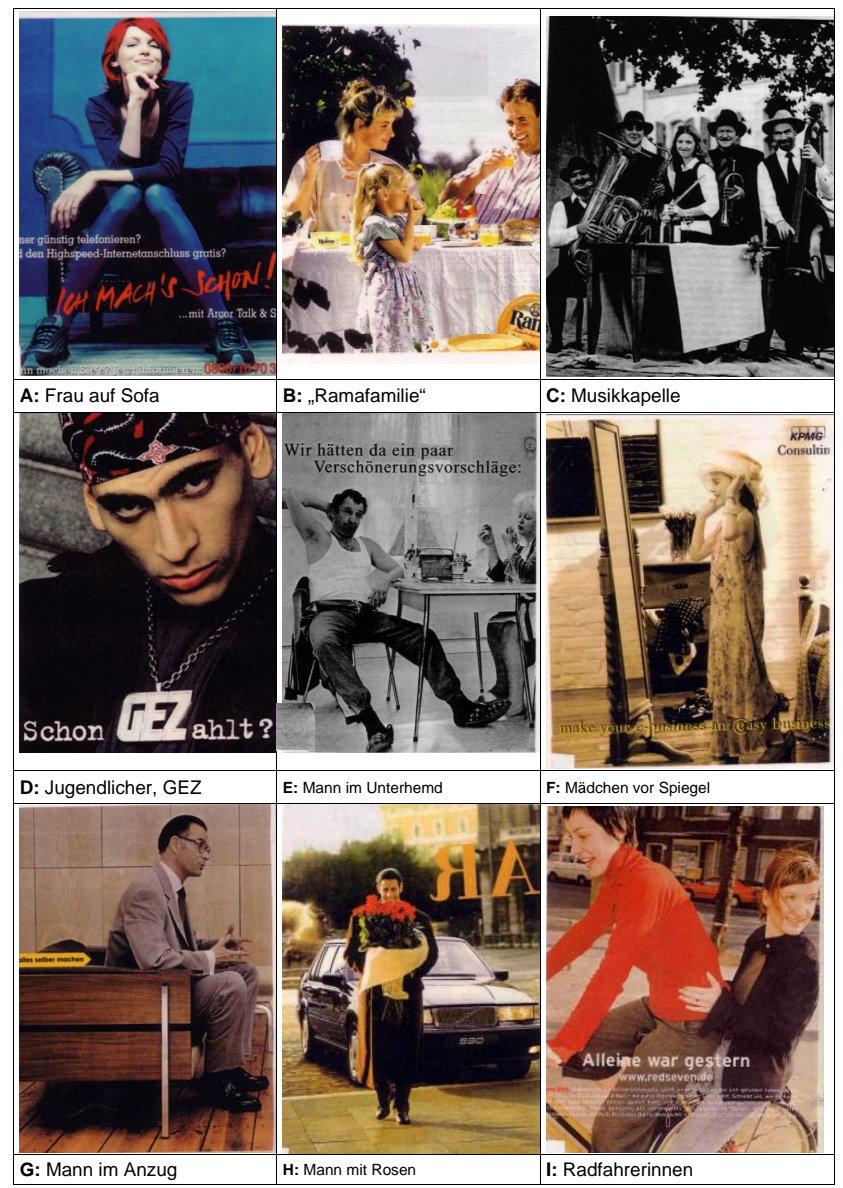

Abb. 1. Zielgruppenorientierte Werbung für Bildassoziationsverfahren. Quellen: Der Spiegel vom 8 Mai, 15 Mai, 22 Mai 2000 und 29 Mai 2000, Der Stern vom 19 April 2000, Prinz vom 28 April bis 31 Mai 2000.
SGD

3, 183-210, 2007

\section{Raumbezogene} Vorstellungsbilder, visuelle Methodik

K. Wiest

Title Page

Abstract Introduction

Conclusions References

Tables Figures

IS

4

>

Back

Close

Full Screen / Esc

Printer-friendly Version

Interactive Discussion

Copernicus 


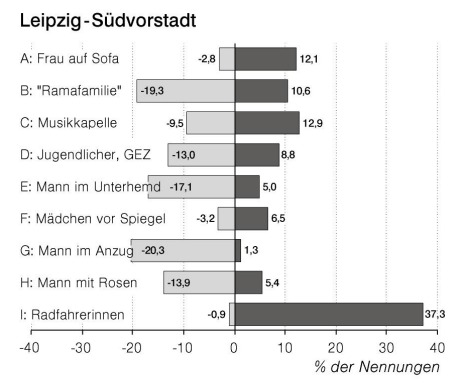

Leipzig-Waldstraßenviertel

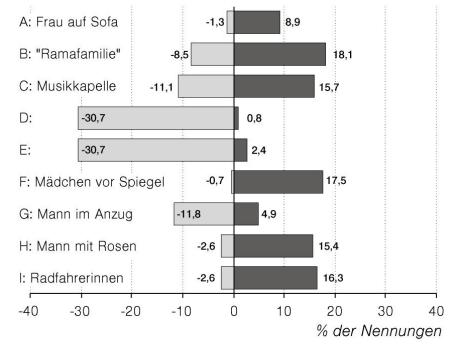

Leipzig-Neustädter Markt

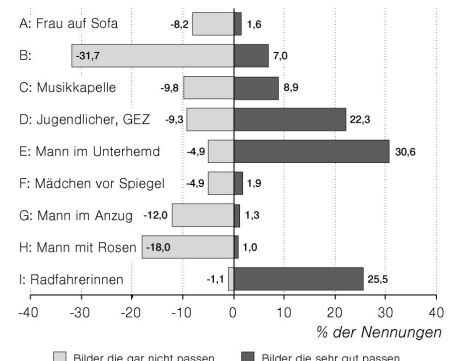

Pan

Abb. 2. Ergebnisse der Haushaltsbefragung: Welche Bilder passen zu Ihrem Wohnquartier? ( $N=276$ (Südvorstadt), 276 (Waldstraßenviertel), 206 (Neustädter Markt)
SGD

3, 183-210, 2007

\section{Raumbezogene}

Vorstellungsbilder,

visuelle Methodik

K. Wiest

Title Page

Abstract

Introduction

Conclusions

References

Tables

\section{Figures}

14

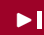

4

Back

Close

Full Screen / Esc

Printer-friendly Version

Interactive Discussion

Copernicus 
SGD

$3,183-210,2007$

Raumbezogene Vorstellungsbilder, visuelle Methodik

K. Wiest

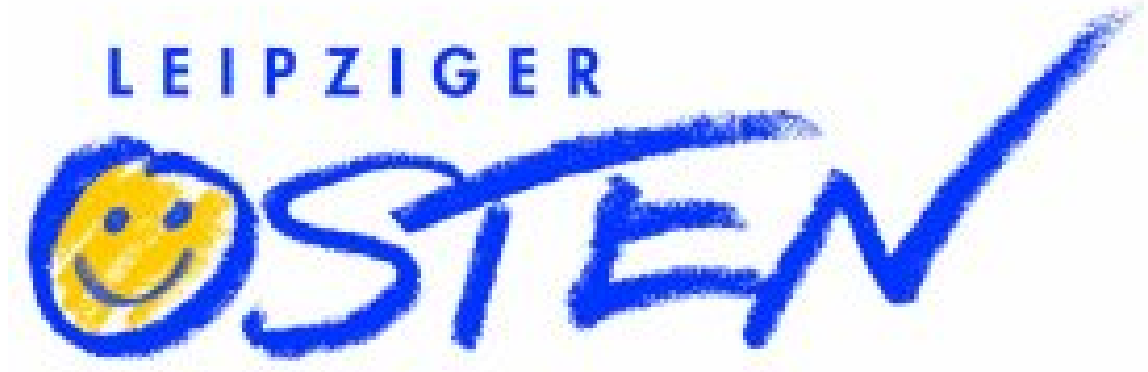

Im Osten geht die Sonne auf.

Abb. 3. Das Logo des Leipziger Ostens (Programmgebiet Soziale Stadt). Quelle: http://www. leipziger-osten.de

\begin{tabular}{|c|c|}
\hline \multicolumn{2}{|c}{ K. Wiest } \\
\hline \multicolumn{2}{|c|}{ Title Page } \\
\hline Abstract & Introduction \\
\hline Conclusions & References \\
\hline Tables & Figures \\
\hline I4 & \\
\hline 4 & \\
\hline Back & \multicolumn{1}{c}{ Close } \\
\hline Full Screen / Esc
\end{tabular}

Printer-friendly Version

Interactive Discussion 